\title{
An Analysis of Consumer Protection for Gamblers Across Different Online Gambling Operators in Ireland: A Descriptive Study
}

\author{
Caoimhe Cooney ${ }^{1} \cdot$ David Columb $^{2} \cdot$ Joao Costa $^{2} \cdot$ Mark D. Griffiths $^{3}$ (D) \\ Colin $\mathrm{O}^{\prime}$ Gara $^{4,5}$
}

Published online: 18 September 2018

(C) The Author(s) 2018

\begin{abstract}
The aim of the present study was to evaluate the responsible gambling tools which are available to online gamblers at Irish online gambling websites. The present study used a similar methodology to a recent study carried out on the world's most popular websites (Bonello and Griffiths Gaming Law Review and Economics, 21, 278-285, 2017), where 50 of the most advertised online gambling websites were evaluated in relation to their responsible gambling (RG) practices. The present study evaluated 39 gambling websites with either a ".ie" or ".com/ie" domain. Each website was evaluated by checking for a number of RG practices, including presence of a dedicated RG page; age verification; access to gambling account history; the availability of RG tools, such as limit setting facilities and exclusion settings; and links to limit-setting options on the deposit page. Descriptive statistics were then performed on the results from each website. Of the 39 online gambling operators identified, 22 redirected gamblers to a ".com" domain, while 17 operators remained as a ".ie" domain. Thirty-five websites $(89.7 \%$ ) visited had a dedicated RG page. Responsible gambling features were evaluated and demonstrated to be available in an inconsistent manner across online gambling websites. Irish websites were shown to perform poorly in comparison with non-Irish counterparts in the provision of RG tools. The researchers of the present study are not aware of any similar studies conducted to date in Ireland.
\end{abstract}

Keywords Gambling · Responsible gambling · Problem gambling · Online gambling · Responsible gambling tools $\cdot$ Gambling websites

Most people have gambled at some point during their lives (Calado and Griffiths 2016). Data relating to consumer trends published by the UK Gambling Commission in 2017 reported that $45 \%$ of people had gambled over the preceding four weeks, and that $18 \%$ of people had gambled

Mark D. Griffiths

mark.griffiths@ntu.ac.uk

Extended author information available on the last page of the article 
online over the preceding four weeks (Government of UK-Gambling Commission 2018). Figures from Northern Ireland (NI) suggest that there is a $67.2 \%$ past year prevalence for gambling activities, with a rate of $2.3 \%$ for problem gambling (Government of Northern Ireland 2017). A need has been identified for critical survey work to be done in Ireland (Irish Medical Organisation 2015; O'Gara 2017), because there is a dearth of academic studies which have adequately investigated problem gambling in Ireland to date. The National Advisory Committee on Drugs and Alcohol (NACDA) began recording figures for gambling in Ireland in 2014 and reported a gambling prevalence rate of 64.5\% during 2014/2015 in the Irish adult population, although this represented a broad definition of gambling, including the National Lottery (Government of Ireland 2016). Griffiths identified a relatively high gambling propensity among the Irish population compared to other European countries based on Gross Gambling Revenue (Griffiths 2010).

Problem gambling is also a current topic of conversation in Irish legislature, particularly given the recent approval from the Irish government to introduce updated legislation in the form of the Gambling Control Bill 2013, which is due to supersede the outdated Gaming and Lotteries Act 1956 in Ireland (Government of Ireland, Gambling Control Bill 2013). There has been a rise in remote gambling, including online gambling, in recent years. Recent figures from the Gambling Commission in the UK indicate that online gambling accounts for $33 \%$ of all gambling, and now represents the largest gambling sector in the UK (Gambling Commission 2018), although technically, online gambling is a medium and comprises of many different types of gambling. As online gambling participation increases, it is likely that gambling-related harm will also continue to increase (Gainsbury and Wood 2011). Specific characteristics particular to online gambling, such as increased affordability, accessibility, anonymity, and convenience may elevate the risk posed to more vulnerable gamblers, such as adolescents, the intoxicated, and problem gamblers. (McCormack and Griffiths 2012). It has been suggested that the medium of the Internet may be more likely to contribute to problem gambling than gambling in offline environments for those that are already vulnerable or susceptible (Griffiths et al. 2009a).

Further research in this area has outlined that the situation is more nuanced because most online gamblers also gamble offline (Wardle et al. 2011). Gamblers who engage in mixed modes of gambling both online and offline are most at risk of problem gambling compared to those who only gamble online or only gamble offline (Wardle et al. 2011). An online survey carried out by Columb and O'Gara (2017) reported that online gamblers in Ireland demonstrate similar behavioural profiles to online gamblers in the UK and worldwide with a similar burden of financial and mental health sequelae. The relationship between electronic gambling and problem gambling is unclear and may be influenced by structural characteristics of specific games (Wood and Griffiths 2015). An argument has also been put forward that the same technological advancements utilised in online gambling may also be used to provide responsible gambling tools and solutions to reduce gambling-related harm (Harris and Griffiths 2017). Furthermore, it has been suggested that a lack of regulatory standards online leaves online gamblers vulnerable without adequate consumer protection, and this was highlighted by Gainsbury et al. (2013) who found that around one-third of online gamblers have disputes with online gambling sites.

While there are limitations to the current evidence base for responsible gambling tools, a recent review indicated that these tools are best viewed as a responsible gambling prevention measure for those who are still gambling within safe limits rather than for problem gamblers, although problem gamblers should continue to be included in ongoing research efforts in this area (Harris and Griffiths 2017). Ladouceur et al. (2017) indicated that there is some evidence 
to suggest particular areas, which provide the opportunity to inform best practice in the area of harm minimisation tools, including self-exclusion, limit setting, responsible gambling machine features, and employees training. Griffiths et al. (2009b) identified that many responsible gambling features were viewed as being useful by gamblers themselves, including limit setting, viewing their gambling profile, self-exclusion facilities, self-diagnostic problem gambling tests, information and support for gambling issues, and gambling profile prediction.

Given the importance of responsible gambling practices in online gambling, the aim of the present study was to evaluate the responsible gambling tools which are available to online gamblers at Irish online gambling websites. The study used a similar methodology to a recent study carried out on the world's most popular websites (Bonello and Griffiths 2017), where 50 of the most advertised online gambling websites were evaluated in relation to their responsible gambling practices.

\section{Methods}

The present study evaluated 39 gambling websites with either a '.ie' or '.com/ie' domain. The selection for the gambling operators chosen was carried out via different online search engines (Google and Yahoo), using the search terms 'gambling', 'betting', 'poker', 'poker online', 'casino', and 'casino online'. The search was performed using a standard search format and then was further filtered by domain. Each online gambling operator's website was evaluated in further detail by checking for responsible gambling practices (listed below). The responsible gambling practices evaluated were adapted from the study conducted in the UK by Bonello and Griffiths (2017). More specifically, the following responsible gambling practices were examined as to whether the online gambling website had any of the following:

A. A dedicated RG page including the following criteria:

1. A statement on the operator's commitment to RG

2. A warning that gambling can be harmful

3. Reference to a problem gambling help organisation and/or self-help groups

4. A self-assessment test for problem gambling

5. Links to gambling filtering software such as GamBlock and/or Betfilter

6. Links to age filtering software

B. Initial age checks during the account registration phase

C. A link to the RG page or information about RG practices in the communication sent by the operator to those registering to gamble on the website

D. The presence and easy accessibility of gambling account history

E. The availability of RG tools including:

1. Limit setting facilities

- Deposit limit

- Spending limit

- Loss limit

- Session limit 
2. Exclusion settings

- Cooling-off periods

- Voluntary self-exclusion

3. Other RG tools

- Reality checks

- Animated educational videos

- Gambling diary

- Budget calculator

F) A link to limit-setting option on the deposit page

Descriptive statistics were then performed on the results from each website. A further comparison was then drawn between the websites that remained in their Irish domain (.ie or .com/ie) and those that were originally Irish (.ie or .com/ie) in the Internet search but linked out to a .com (non-Irish) domain.

\section{Results}

Of the 39 online gambling operators identified, 22 redirected gamblers to a '.com' domain, while 17 operators remained as a '.ie' domain. Therefore, in addition to the evaluation of each of the RG practices previously mentioned, the performance of the '.ie' domain operators were compared against the '.com' operators. The websites that remained '.ie' and '.com/ie' websites will be referred to as Irish websites, while the websites that linked out to a '.com' website will be referred to as non-Irish websites.

\section{Dedicated Responsible Gambling Page}

A total of 35 websites $(89.7 \%$ ) visited had a dedicated responsible gambling page. Of the 22 non-Irish online gambling operators examined, the large majority provided for a link for gamblers to an RG page $(n=20,90.9 \%)$. However, on one occasion, it was listed as 'information' rather than RG. In another instance, the link to RG page was not easily accessible, meaning gamblers had to search for it indirectly, using a search engine, such as Google. Fifteen of the 17 Irish operators had a link to a dedicated RG page. One of the operators did not have a RG page, while for another gambling operator, the responsible gambling page was available on its parent website but not on the actual gambling operator's website. Of the 35 online gambling operators that had a dedicated RG page, all had a warning that gambling can be harmful and all had a statement concerning their commitment to RG.

\section{Reference to Problem Gambling Organisations}

Most operators $(n=37)$ referenced at least one organisation where users could get help for a gambling problem $(94.9 \%)$. The two operators which did not provide reference to a 
problem gambling help organisation and/or self-help groups were both Irish domain websites.

\section{Responsible Gambling Software}

Approximately half of the gambling operators $(n=18 ; 46.2 \%)$ provided links to gambling filtering software, such as Betfilter or Gamblock to block access to online gambling websites, of which ten were non-Irish (45.5\%) and eight were Irish domain operators (47.1\%). Threequarters of the operators $(74.7 \%)$ mentioned or provided links to age filtering software, including Netnanny, Cybersitter, and/or Cyberpatrol. Eighteen of those were non-Irish domain websites $(81.8 \%)$, while 11 were Irish domain websites $(64.7 \%)$.

\section{Self-Assessment Tools}

Most operators $(n=27)$ displayed a problem gambling self-assessment tool $(69.2 \%)$, either as a checklist or as an interactive quiz. Of the 27 gambling operators with a self-assessment tool, 17 were non-Irish (72.7\%), while 11 were Irish domain operators (64.7\%). A checklist format was used on 17 websites $(63.0 \%)$. The interactive format was offered on nine websites $(33.3 \%)$, with one website offering both formats to users.

\section{Age Verification Check on Account Registration}

Of the 39 online gambling operators, all of them (100\%) required individuals to be 18 years of age or over and, would not allow a date of birth under 18 years old to be entered when gamblers registered for an account. In addition, all operators (100\%) required a phone number, a minority of which $(n=6)$ sent an authorisation code to the gamblers phone $(15.4 \%)$. Most gambling operators $(n=37)$ did not require identity documents to confirm the age of the registrants $(94.9 \%)$. One operator required further documents (bank/credit card statement or recent utility bill) as part of its setup and, another operator looked for additional age verification (bank/credit card statement or recent utility bill) prior to entering site, although the reason for this concerned possible fraudulent behaviour on the account, rather than being age-related.

\section{Responsible Gambling Information via Email}

Out of the 39 gambling operators, three were excluded in the evaluation of this RG practice, either due to difficulties signing up (registration option malfunctioned, despite a number of attempts to register), or because of age verification requirements (requesting proof of address via bank/credit card statement or recent utility bill). Three-quarters of the 36 remaining gambling operators examined $(n=27)$ sent a welcome email after registration to the players $(75 \%)$. In a very small number of cases $(n=3)$, although gamblers did not receive a welcome email, they received other emails including notifications of cooling-off periods (8.33\%). Of the 22 gambling operators that sent a welcome email, approximately half of them $(n=13)$ mentioned RG in the email sent (48.1\%). Of these, eight of out 15 were non-Irish operators $(53.3 \%)$ and 5 out of 12 were Irish domain operators (41.6\%). Approximately two-thirds of the gambling operators $(n=$ 17) encouraged depositing funds in the welcome email sent (63\%), of which 9 out of 15 were non-Irish domain websites (60\%) and 8 out of 12 were Irish operators $(66.7 \%)$. 


\section{Access to Gambling Account History}

Of the 39 gambling operators included in this study, three of them (7.6\%) were not evaluated for the presence and accessibility of gambling account history, either due technical issues or because (in one instance) the customer was blocked for further verification. However, of the 36 operators examined, the vast majority $(n=35)$ offered an option for gamblers to access their account history $(97.2 \%)$.

\section{Responsible Gambling Tools Offered by Website}

Almost all of the gambling operators visited online $(n=38)$ offered some form of responsible gambling tool with their service $(97.4 \%)$. Of note, the one operator that did not offer any responsible gambling service was listed as a '.ie' website but linked out to a '.com' website, where it stated that it did not accept any business from a number of countries including Ireland. A total of 35 of the online gambling operators $(89.7 \%)$ offered limit-setting tools with their service. Of the non-Irish sites, 21 offered limit-setting tools (95.5\%) compared to the remaining 14 Irish-based sites (82.4\%). Of these limits, deposit limits were the most frequently offered, with 33 out of 35 websites offering them to their customers (94.3\%). Out of the 22 non-Irish operators, only two operators provided spending limits on specific activities, while only three operators offered loss limits on their casino games and not on all games/activities offered on their websites. Approximately one-third of the gambling operators surveyed $(n=$ 12) offered a single limit-setting option (34.3\%). Seven of the operators had the option to set two limits $(20 \%)$, while nine websites offered three limits $(25.7 \%)$. Only two operators provided gamblers with four limit-setting options (5.7\%).

\section{Link to Limit-Setting on Deposit Page}

Of the online gambling sites examined, 22 of them provided a limit-setting option for gamblers on the deposit page $(57.9 \%)$. Approximately half of the Irish gambling operators examined $(n=9)$ provided a link to a limit-setting option on the deposit page $(52.9 \%)$. Of the non-Irish operators, most of them $(n=15)$ offered a limit-setting option on the deposit page $(71.4 \%)$. However, two of those operators merely provided a link to minimum deposit amounts despite being described as deposit limits (Tables 1 and 2 ).

\section{Cooling-Off and Self-Exclusion Periods}

Cooling-off periods refer to any period of exclusion from gambling on the operator's website for less than six months (following the definition used in the study by Bonello and Griffiths 2017). A total of 25 websites offered cooling-off periods via the gambling website only $(64.1 \%)$, with 16 of these websites being non-Irish (72.7\%) and nine Irish (52.9\%). Further,

Table 1 Limit-setting tools offered by gambling websites surveyed

\begin{tabular}{lcrr}
\hline & Irish $(n=14)$ & Non-Irish $(n=21)$ & Total $(n=35)$ \\
\hline Deposit limit & $14(100.0 \%)$ & $19(90.5 \%)$ & $33(94.3 \%)$ \\
Spending limit & $5(35.7 \%)$ & $7(33.3 \%)$ & $12(34.3 \%)$ \\
Loss limit & $2(14.3 \%)$ & $12(57.1 \%)$ & $14(40.0 \%)$ \\
Session limit & $3(21.4 \%)$ & $4(19.0 \%)$ & $7(20.0 \%)$ \\
\hline
\end{tabular}


Table 2 Multiple limit settings offered by gambling websites

\begin{tabular}{lllr}
\hline & Irish $(n=14)$ & Non-Irish $(n=21)$ & Total $(n=35)$ \\
\hline One limit & $9(64.3 \%)$ & $3(14.3 \%)$ & $12(34.3 \%)$ \\
Two limits & $1(7.1 \%)$ & $6(28.6 \%)$ & $7(20.0 \%)$ \\
Three limits & $3(21.4 \%)$ & $6(28.6 \%)$ & $9(25.7 \%)$ \\
Four limits & $1(7.1 \%)$ & $1(4.8 \%)$ & $2(5.7 \%)$ \\
\hline
\end{tabular}

six websites (three Irish, three non-Irish) required customers to contact customer services in order to activate the cooling-off period. This brought the total number of websites offering a cooling-off period to 33 (84.6\%), of which 20 were non-Irish (90.9\%) and 13 Irish (76.5\%).

On a few occasions, gambling operators allowed cooling-off periods significantly shorter than six months. One non-Irish operator only allowed a cooling-off period of up to one week, and one Irish website offered a cooling-off period of up to six weeks. In one other instance, the cooling-off period could not be activated until real money had been deposited. Self-exclusion periods refer to any period of exclusion from gambling on the operator's website for more than six months (Bonello and Griffiths 2017). A total of 23 websites offered self-exclusion periods via the website only (59.0\%), with 13 of these websites being non-Irish (59.1\%) and being 10 Irish (58.8\%). A further 15 websites (six Irish, nine non-Irish) required the individual to contact customer services in order to activate the self-exclusion period. This brings the total number of websites offering a self-exclusion period to $38(97.4 \%)$, comprising 22 non-Irish (100\%) and 16 Irish $(94.1 \%)$ (Table 3).

\section{Other Responsible Gambling Tools}

Twenty operators offered session-time reminders (51.3\%), most often termed 'reality checks'. These session-time reminders were offered by 12 non-Irish websites $(54.4 \%)$ and eight Irish websites $(47.1 \%)$. Of the Irish operators, only one offered reality checks for some gambling games, such as casino and poker. Of the non-Irish operators, only one offered reality checks for some gambling games except for poker. Other types of RG tools offered by the gambling websites visited included animated educational videos (two websites; 5.1\%), a gambling diary (three websites; 7.7\%) and a budget calculator (three websites; 7.7\%). Two websites, one nonIrish and one Irish, offered forms for permanent offline store exclusion, while three operators offered permanent exclusion, two of which were Irish operators.

Table 3 Exclusion settings and other responsible gambling tools offered by gambling websites surveyed

Irish $(n=17)$ Non-Irish $(n=22)$ Total $(n=39)$

\begin{tabular}{|c|c|c|c|}
\hline \multicolumn{4}{|l|}{ Exclusion settings } \\
\hline Cooling-off periods (via website) & $9(52.9 \%)$ & $16(72.7 \%)$ & $25(64.1 \%)$ \\
\hline Cooling-off periods (via website/customer service contact) & $13(76.5 \%)$ & $20(90.9 \%)$ & $33(84.6 \%)$ \\
\hline Voluntary self-exclusion (via website) & $10(58.8 \%)$ & $13(59.1 \%)$ & $23(59.0 \%)$ \\
\hline $\begin{array}{l}\text { Voluntary-self exclusion (via website/customer } \\
\text { service contact) }\end{array}$ & $16(94.1 \%)$ & $22(100.0 \%)$ & $38(97.4 \%)$ \\
\hline \multicolumn{4}{|l|}{ Other responsible gambling tools } \\
\hline Reality checks/session time reminders & $8(47.1 \%)$ & $12(54.5 \%)$ & $20(51.3 \%)$ \\
\hline Animated educational videos & $0(0.0 \%)$ & $2(9.1 \%)$ & $2(5.1 \%)$ \\
\hline Gambling diary & $1(5.9 \%)$ & $2(9.1 \%)$ & $3(7.7 \%)$ \\
\hline Budget calculator & $1(5.9 \%)$ & $2(9.1 \%)$ & $3(7.7 \%)$ \\
\hline
\end{tabular}




\section{Discussion}

The aim of the present study was to evaluate the responsible gambling tools, which are available to online gamblers from Irish gambling websites. The study's methodology was adapted to Irish online gambling websites from a study previously conducted by Bonello and Griffiths (2017). The findings of the study by Bonello and Griffiths (2017) reflects previous research in the UK, which has identified a lack of consistent consumer protection on online gambling websites used by UK based customers (e.g., Khazaal et al. 2013; Smeaton and Griffiths 2004). The research team of the present study are not aware of studies of a similar nature conducted to date in Ireland. The main findings of the present study demonstrated that the vast majority of online operators (both 'non-Irish' and 'Irish' websites) have a dedicated responsible gambling page and that the majority of online gambling websites offer some types of responsible gambling tools to help minimise harm from problem gambling.

In relation to having a dedicated responsible gambling page, the results here were similar to previous studies completed in this area. In the study by Bonello and Griffiths (2017), every website visited had a dedicated responsible gambling page, whereas this study found approximately $90 \%$ of websites had one. A key reason for this difference may be that the Bonello and Griffiths (2017) study surveyed the top 50 most advertised websites via a search engine. In the present study, the research attempted to identify as many '.ie' websites as possible, thus including less well-known websites, with the possibility of less stringent adherence to responsible gambling. The content of the responsible gambling pages was also evaluated. The evaluation broadly looked for information on (i) where to seek help for problem gambling, (ii) how individuals can identify problem gambling behaviours in themselves, and (iii) referral to organisations that help reduce the harm from gambling online.

The vast majority of websites referenced at least one organisation where individuals with a gambling problem could seek help (94.9\%). This is consistent with the study by Bonello and Griffiths (2017), which reported that $84 \%$ of the gambling websites visited offered some information on help for problem gambling. A study published in 2004 by Smeaton and Griffiths reported that only $13.3 \%$ of UK websites visited at the time offered such information. The findings of the present study and the study by Bonello and Griffiths (2017) appear to demonstrate that there has been a marked improvement in awareness by online gambling operators of the need for player protection within the UK over the past 15 years. It is not possible to determine whether there has been any change in the provision of consumer protection measures on online gambling websites in Ireland because the research team of the present study are not aware of similar research previously conducted in Ireland.

Self-assessment tools were offered by approximately two-thirds of operators in the present study (69.2\%), similar to the findings of Bonello and Griffiths' (2017) study (64.0\%). Irish websites performed less well compared to their non-Irish counterparts in this regard, with $72.7 \%$ of non-Irish websites offering self-assessment checks compared to $64.7 \%$ of Irish websites. There was also some variation in the way in which self-assessment tools were offered. Approximately, two-thirds of gambling websites offered a checklist format compared to one-third offering an interactive format. Interactive interventions likely offer better outcomes (Shahab and McEwen 2009), and the increased use of interactive checklists may help promote awareness of problem gambling behaviour among online gambling website clientele.

Information on software to restrict access to gambling was subdivided in the present study to software that filters out online gambling websites (Gamblock, Betfilter), and software that restricts underage gambling on online gambling websites (e.g., Netnanny, Cyberpatrol, and 
Cybersitter). Just under half of websites visited offered information on gambling website filtering software $(46.2 \%$ ), which was less than the $60 \%$ reported by Bonello and Griffiths (2017). A larger number of online gambling websites offered information in relation to age filtering software (74.7\%) compared to gambling filtering software. There was a large difference between Irish and non-Irish websites visited, with the Irish websites $(64.7 \%)$ performing more poorly compared to non-Irish $(81.8 \%)$ websites. This suggests gambling operators focus more on preventing underage gambling on their websites than restricting access to gambling websites.

This was also highlighted with all websites visited requiring their users to be aged 18 years or over. This is in contrast to Bonello and Griffiths (2017), which reported around two-thirds of gambling operators offered a prominent display of needing to be 18 -years-old to participate. The difference in these findings may be due to different methodology employed in the present study. In the present study, the research team assessed whether or not an under-age date of birth could be entered, which none of the websites visited allowed. One Irish website did require identity documents as part of its registration phase, and was one of the few areas in which Irish websites outperformed their non-Irish counterparts in relation to responsible gambling. This is an area where improvement is urgently required, because there is a lot of empirical evidence highlighting the adverse effects that gambling can have on adolescents, as well as the prevalence of gambling being higher among the adolescent population in comparison to adults (Calado et al. 2017; Gupta and Derevensky 2000; Molinaro et al. 2014; Volberg et al. 2010).

\section{Responsible Gambling Tools}

A total of 38 out of 39 of the online gambling websites visited (97.4\%) offered some form of responsible gambling tool with their service. This is comparable with the Bonello and Griffiths study, which demonstrated that most online gambling operators had RG tools, such as limitsetting ( $n=45 ; 90 \%)$, cooling-off periods $(n=36 ; 72 \%)$, and voluntary self-exclusion $(n=43$; $86 \%)$. Research consistently indicates that gamblers frequently gamble more than intended (Ladouceur et al. 2012). Setting predetermined limits, prior to engaging in gambling, can facilitate gamblers to gamble within their means, by preventing unintended and impulsive transactions occurring as the gambling session progresses (Scannell et al. 2000). In the present study, 35 of the online gambling sites $(89.7 \%)$ offered limit-setting tools with their service. This is in line with findings from Bonello and Griffiths (2017), where 90\% of the online gambling websites evaluated offered limit-setting facilities. Of the non-Irish gambling websites, 21 offered limit-setting tools (95.5\%) compared to 14 of Irish-based sites (82.4\%). Of these limit-setting tools, deposit limits were the ones most frequently offered, with 33 out of 35 websites offering these to their customers (94.3\%).

Spending limits were offered on a restricted number of games on $34 \%$ of online gambling websites. Loss limits were offered by $40 \%$ of gambling websites and $20 \%$ of sites offered session limits. There is empirical evidence that suggests that limit setting can be effective in promoting RG for some individual (Auer and Griffiths 2013). A study carried out by the International Gaming Research Unit comprising 10,865 online gamblers in 2007 found that $70 \%$ of players thought that voluntary spending limits would be beneficial (cf. Griffiths et al. 2011). This finding was replicated in a 2009 study by Griffiths et al. (2009b) examining the attitudes and behaviours of 2348 Swedish online gamblers, $70 \%$ of whom reported that spending limits were a useful responsible gambling tool. A study examining a sample of 100,000 online gamblers playing on the win2day gambling website showed decreased spending and playing time among poker players after setting a voluntary time limit, with the biggest effect observed among the highest intensity 
gamblers (Auer and Griffiths 2013). A study by Kim et al. (2014) suggested that the use of time limits is an effective responsible gambling tool. Here, gamblers who were reminded to consider using a time limit tool spent considerably less time gambling than those who did not receive this reminder. A study by Hing et al. (2015a) also emphasised the tendency of regular gamblers to go beyond self-imposed limits, which suggests that online gamblers would benefit from limit-setting mechanisms imposed by online gambling websites.

Reality checks have been posited as a mechanism for interrupting gambling or gaming to allow the player time to reflect to their actions (Harris and Griffiths 2017). This may take the form of session-time reminders or an in-built pause every 20 min during play. Reality checks were offered by one Irish website but only for two games (i.e., casino and poker), and by one non-Irish website only. Pop-up messages were not observed on any of the Irish websites visited. Auer and colleagues (Auer and Griffiths 2015a; Auer et al. 2014) demonstrated in two studies that there is some evidence for a very small increase in the number of gamblers discontinuing their session of play following pop-up messages. They also noted that messages containing more sophisticated data displaying normative and self-appraisal content were a little more effective than simple messages (Auer and Griffiths 2015b).

Cooling-off periods have been claimed to be a useful responsible gambling tool to facilitate a period of time where the player can reflect on their gambling behaviour, with the aim of reducing compulsive gambling and chasing financial losses (Williams et al. 2012). However, there is a lack of consensus about the frequency and duration in breaks of play, which would provide most robust protection to the gambling population (Blaszczynski et al., 2016). Cooling-off periods were defined as a period of less than six months of duration by Bonello and Griffiths (2017). In the present study, seven online gambling websites did not allow a cooling-off period without first contacting customer services. The cooling-off period was less than six months on four online gambling websites. On one online gambling website, the cooling-off period could not be activated until real money had been deposited, which reflected the findings from Bonello and Griffiths (2017), where $72 \%$ of websites visited provided a cooling-off period.

Cooling-off periods have previously been identified as a best practice option that should exist for any user to exclude themselves from a site, and that the procedure for self-exclusion should be clearly stated in the rules and procedures page, with a minimum exclusion period of six months (Smeaton and Griffiths 2004). Hing et al. (2015b) conducted a study demonstrating that selfexclusion provides an important harm reduction option with short-term benefits for many participants. While in most cases in the present study the option of self-exclusion was available (100\% of non-Irish websites and $94.1 \%$ of Irish websites), it was necessary in over $40 \%$ of cases to contact customer services for this to be enacted. These rates are higher than those reported by Bonello and Griffiths (2017), in which $86 \%$ of evaluated websites provided voluntary self-exclusion periods. In the present study, only three online gambling websites visited offered permanent self-exclusion.

There were a small number of additional RG features offered by some Irish gambling websites. A gambling diary was offered by three gambling websites. A budget calculator was offered by one Irish gambling website and two non-Irish gambling websites. These appear to be RG initiatives that could be offered by all online gambling websites. It should also be noted that there was a lack of personalised feedback or behavioural tracking tools offered by Irish online gambling websites. Auer and Griffiths (2014, 2015a, 2015b, 2016) demonstrated that personalised behavioural feedback utilizing the 'stages of change' model (Prochaska et al. 1992) and the principles of motivational interviewing can be effective in promoting more responsible gambling. The introduction of robust behavioural feedback provides a potential opportunity for advancement of RG tools in Ireland. 


\section{Conclusion}

As previously noted, the present study was modelled on a previous study by Bonello and Griffiths (2017). The purpose of the present study was to evaluate responsible gambling tools available on online gambling websites used in Ireland by the online gambling community. The findings demonstrate that responsible gambling tools are provided in an inconsistent manner across gambling websites, with varying degrees of adherence to what is currently considered to be best practice in the provision of responsible gambling online. The present study benefited from an extensive internet search resulting in a low likelihood of undetected websites. The evaluation of Irish and non-Irish domain websites allowed an in-study comparison of RG tools available between countries, and which provided an extra dimension to the present investigation.

However, the present study is not without limitations. While efforts were made to conduct an extensive Internet search of all websites, the total number of websites amounted to only 39 . No real money was exchanged during the visiting and evaluation of online gambling websites; therefore, it was difficult for the research team to ascertain the true gambling experience from the perspective of an actual online gambler. The present study was also limited by a lack of longitudinal assessment of websites (i.e., each online gambling website was visited only once). However, the possibility remains of evaluating these online gambling websites over a longer time frame in future studies. There is clear scope for improvement in the provision of responsible gambling features available to Irish online gamblers, particularly in the area of behavioural feedback tools, age verification, and limit-setting, to facilitate robust consumer protection within the online gambling community.

\section{Compliance with Ethical Standards}

Conflict of Interest MG's university currently receives funding from Norsk Tipping (the gambling operator owned by the Norwegian Government) for ongoing research. MG has received funding for a number of research projects in the area of gambling education for young people, social responsibility in gambling and gambling treatment from Gamble Aware (formerly the Responsibility in Gambling Trust), a charitable body, which funds its research program based on donations from the gambling industry. MG also undertakes consultancy for various gaming companies in the area of social responsibility in gambling.

The remaining authors (CC, DC, JC, and COG) have no conflicts of interest.

Open Access This article is distributed under the terms of the Creative Commons Attribution 4.0 International License (http://creativecommons.org/licenses/by/4.0/), which permits unrestricted use, distribution, and reproduction in any medium, provided you give appropriate credit to the original author(s) and the source, provide a link to the Creative Commons license, and indicate if changes were made.

\section{References}

Auer, M., \& Griffiths, M. D. (2013). Voluntary limit setting and player choice in most intense online gamblers: an empirical study of gambling behaviour. Journal of Gambling Studies, 29, 647-660.

Auer, M., \& Griffiths, M. D. (2014). Personalised feedback in the promotion of responsible gambling: a brief overview. Responsible Gambling Review, 1, 27-36.

Auer, M. M., \& Griffiths, M. D. (2015a). Testing normative and self-appraisal feedback in an online slot-machine pop-up in a real-world setting. Frontiers in Psychology, 6, 339.

Auer, M., \& Griffiths, M. D. (2015b). The use of personalized behavioral feedback for problematic online gamblers: an empirical study. Frontiers in Psychology, 6, 1406.

Auer, M., \& Griffiths, M. D. (2016). Personalized behavioral feedback for online gamblers: a real world empirical study. Frontiers in Psychology, 7, 1875. 
Auer, M., Malischnig, D., \& Griffiths, M. D. (2014). Is 'pop-up' messaging in online slot machine gambling effective? An empirical research note. Journal of Gambling Issues, 29, 1-10.

Blaszczynski, A., Cowley, E., Anthony, C., \& Hinsley, K. (2016). Breaks in play: Do they achieve intended aims? Journal of Gambling Studies, 32(2), 789-800.

Bonello, M., \& Griffiths, M. D. (2017). Analyzing consumer protection for gamblers across different online gambling operators: a descriptive study. Gaming Law Review and Economics, 21, 278-285.

Calado, F., \& Griffiths, M. D. (2016). Problem gambling worldwide: an update and systematic review of empirical research (2000-2015). Journal of Behavioral Addictions, 5, 592-613.

Calado, F., Alexandre, J., \& Griffiths, M. D. (2017). Prevalence of adolescent problem gambling: a systematic review of recent research. Journal of Gambling Studies, 33, 397-424.

Columb, D., \& O'Gara, C. (2017). A national survey of online gambling behaviours. Irish Journal of Psychological Medicine, 1-9. https://doi.org/10.1017/ipm.2017.64.

Gainsbury, S., \& Wood, R. (2011). Internet gambling policy in critical comparative perspective: the effectiveness of existing regulatory frameworks. International Gambling Studies, 11, 309-323.

Gainsbury, S., Parke, J., \& Suhonen, N. (2013). Consumer attitudes towards Internet gambling: perceptions of responsible gambling policies, consumer protection, and regulation of online gambling sites. Computers in Human Behavior, 29, 235-245.

Government of Ireland - Department of Health, Ireland (2016). Prevalence of Drug Use and Gambling in Ireland and Drug Use in Northern Ireland. Dublin: National Advisory Committee on Drugs and Alcohol.

Government of Ireland - Department of Justice (2013). Gambling Control Bill 2013. Dublin: Department of Justice.

Government of Northern Ireland, Department for Communities, Northern Ireland Statistics and Research Agency (2017). 2016 Northern Ireland Gambling Prevalence Survey. Belfast: Northern Ireland Statistics and Research Agency.

Government of UK, Gambling Commission (2018). Gambling participation in 2017: Behaviour, awareness and attitudes - Annual Report. Birmingham: Gambling Commission.

Griffiths, M. D. (2010). Problem gambling in Europe: what do we know? Casino \& Gaming International, 6, 81-84.

Griffiths, M., Wardle, H., Orford, J., Sproston, K., \& Erens, B. (2009a). Sociodemographic correlates of internet gambling: findings from the 2007 British Gambling Prevalence Survey. Cyberpsychology \& Behavior, 12, 199-202.

Griffiths, M. D., Wood, R. T., \& Parke, J. (2009b). Social responsibility tools in online gambling: a survey of attitudes and behavior among internet gamblers. Cyberpsychology \& Behavior, 12, 413-421.

Griffiths, M., Wardle, H., Orford, J., Sproston, K., \& Erens, B. (2011). Internet gambling, health, smoking and alcohol use: Findings from the 2007 British Gambling Prevalence Survey. International Journal of Mental Health and Addiction, 9, 1-11.

Gupta, R., \& Derevensky, J. L. (2000). Adolescents with gambling problems: From research to treatment. Journal of Gambling Studies, 16, 315-342.

Harris, A., \& Griffiths, M. D. (2017). A critical review of the harm-minimisation tools available for electronic gambling. Journal of Gambling Studies, 33, 187-221.

Hing, N., Cherney, L., Gainsbury, S. M., Lubman, D. I., Wood, R. T., \& Blaszczynski, A. (2015a). Maintaining and losing control during Internet gambling: a qualitative study of gamblers' experiences. New Media \& Society, 17, 1075-1095.

Hing, N., Russell, A., Tolchard, B., \& Nuske, E. (2015b). Are there distinctive outcomes from self-exclusion? An exploratory study comparing gamblers who have self-excluded, received counselling, or both. International Journal of Mental Health and Addiction, 13, 481-496.

Irish Medical Organisation (2015). IMO position paper on addiction and dependency. IMO, 10 Fitzwilliam place, Dublin 2.

Khazaal, Y., Chatton, A., Bouvard, A., Khiari, H., Achab, S., \& Zullino, D. (2013). Internet poker websites and pathological gambling prevention policy. Journal of Gambling Studies, 29, 51-59.

Kim, H. S., Wohl, M. J., Stewart, M. J., Sztainert, T., \& Gainsbury, S. M. (2014). Limit your time, gamble responsibly: setting a time limit (via pop-up message) on an electronic gaming machine reduces time on device. International Gambling Studies, 14, 266-278.

Ladouceur, R., Blaszczynski, A., \& Lalande, D. R. (2012). Pre-commitment in gambling: a review of the empirical evidence. International Gambling Studies, 12(2), 215-230.

Ladouceur, R., Shaffer, P., Blaszczynski, A., \& Shaffer, H. J. (2017). Responsible gambling: a synthesis of the empirical evidence. Addiction Research \& Theory, 25, 225-235.

McCormack, A., \& Griffiths, M. D. (2012). Motivating and inhibiting factors in online gambling behaviour: a grounded theory study. International Journal of Mental Health and Addiction, 10, 39-53.

Molinaro, S., Canale, N., Vieno, A., Lenzi, M., Siciliano, V., Gori, M., \& Santinello, M. (2014). Country-and individual-level determinants of probable problematic gambling in adolescence: a multi-level cross-national comparison. Addiction, 109, 2089-2097. 
O'Gara, C. (2017). The gambling control bill: Time for action. Irish Journal of Psychological Medicine, 1-3. https://doi.org/10.1017/ipm.2017.77.

Prochaska, J. O., DiClemente, C. C., \& Norcross, J. C. (1992). In search of how people change. Applications to addictive behaviors. American Psychologist, 47(9), 1102-1114.

Scannell, E. D., Quirk, M. M., Smith, K., Maddern, R., \& Dickerson, M. (2000). Females' coping styles and control over poker machine gambling. Journal of Gambling Studies, 16, 417-432.

Shahab, L., \& McEwen, A. (2009). Online support for smoking cessation: a systematic review of the literature. Addiction, 104, 1792-1804.

Smeaton, M., \& Griffiths, M. (2004). Internet gambling and social responsibility: an exploratory study. Cyberpsychology \& Behavior, 7, 49-57.

Volberg, R. A., Gupta, R., Griffiths, M. D., Olason, D. T., \& Delfabbro, P. (2010). An international perspective on youth gambling prevalence studies. International Journal of Adolescent Medicine and Health, 22, 3-38.

Wardle, H., Moody, A., Griffiths, M., Orford, J., \& Volberg, R. (2011). Defining the online gambler and patterns of behaviour integration: Evidence from the British Gambling Prevalence Survey 2010. International Gambling Studies, 11, 339-356.

Williams, A. D., Grisham, J. R., Erskine, A., \& Cassedy, E. (2012). Deficits in emotion regulation associated with pathological gambling. British Journal of Clinical Psychology, 51, 223-238.

Wood, R. T. A., \& Griffiths, M. D. (2015). Understanding positive play: an exploration of playing experiences and responsible gambling practices. Journal of Gambling Studies, 31, 1715-1734.

\section{Affiliations}

\section{Caoimhe Cooney $^{1} \cdot$ David Columb $^{2} \cdot$ Joao Costa ${ }^{2} \cdot$ Mark D. Griffiths $^{3} \cdot$ Colin O' Gara $^{4,5}$}

Caoimhe Cooney

Caoimhe.cooney@sjog.ie

David Columb

David.columb@sjog.ie

Joao Costa

Joao.paulo@sjog.ie

Colin O' Gara

Colin.ogara@sjog.ie

1 Department of General Adult Psychiatry, Cluain Mhuire Community Mental Health Service, Newtownpark Avenue, Blackrock, Dublin, Ireland

2 Saint John of God Hospital, Stillorgan, Dublin, Ireland

3 Nottingham Trent University, Nottingham, UK

4 Addictions Department, St. John of God's Hospital, Stillorgan, Dublin, Ireland

5 Medicine and Medical Specialties, UCD School of Medicine, University College Dublin, Dublin, Ireland 